Historic, Archive Document

Do not assume content reflects current scientific knowledge, policies, or practices. 

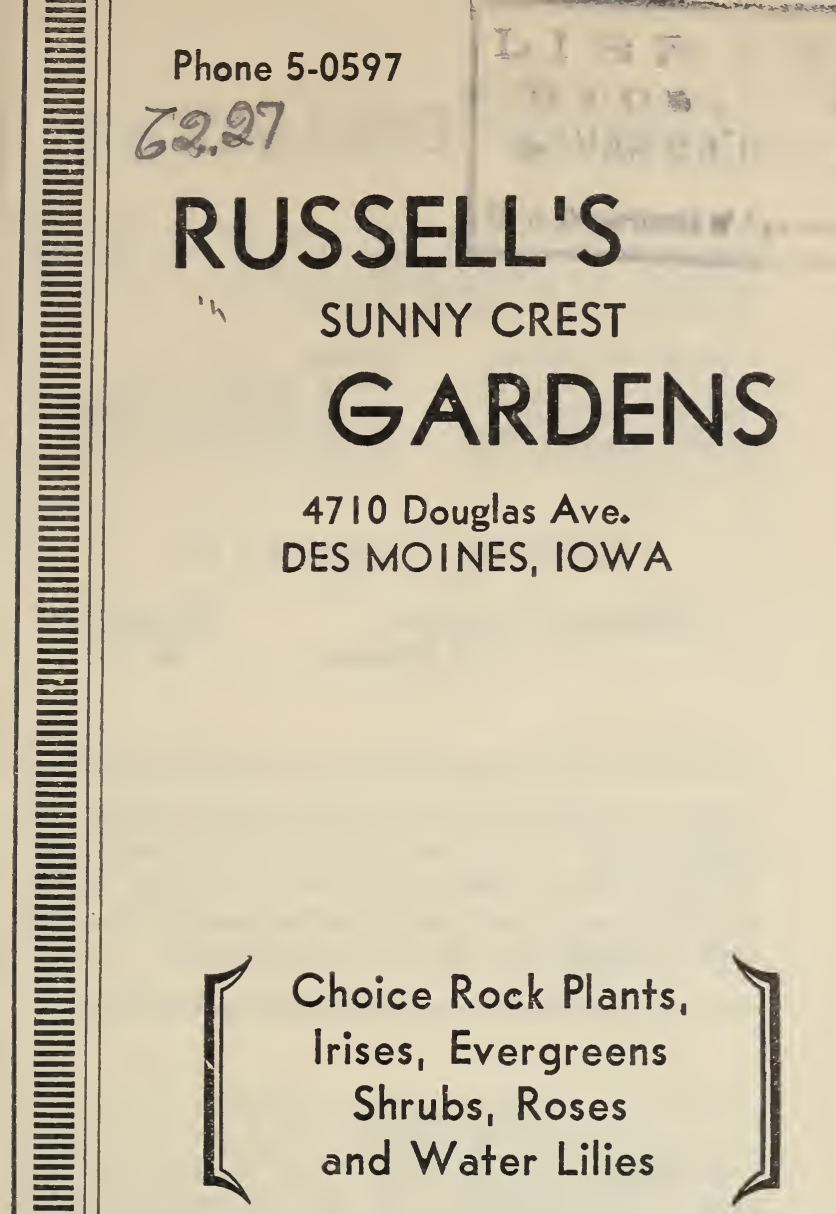

Spring

1932 Shrubs, Roses and Water Lilies 


\section{BANNER COLLECTION}

For those who wish to get that new rock garden planted and are not sure what to plant, we offer the following ten plants, standard stock, well packed and labeled.

Alyssum argenteun Campanula turbinata Dianthus deltoides Linum perenne (Flax) Iris pumilla
Nepeta mussini Phlox Sublata rosea Phlox Sublata lavender Veronica incana Sedum album

\section{Catalog Value $\$ 2.50$}

\section{Collection Price for 10 Plants.

Send in pictures of your rock gardens, pools, and landscapings, as we are interested in seeing how you have worked out your problems with our plants. We will give a $\$ 5.00$ list of hardy plants to the one that is judged the best in late September.

\section{READ CAREFULLY}

All plants offered in this catalog are grown in our fields. All varieties, with few exceptions, which cannot withstand our climatic conditions, are discarded, as we are growers of HARDY Plants. All plants are grown in the open, not greenhouse or hot bed plants, insuring you better success and more vigorous growth in your gardens.

\section{NON-WARANTY}

Because there are so many causes for failure, over which we have no control, such as poor or improper soil, unfavorable situation or careless culture, etc., we can assume no responsibility after stock is delivered.

\section{SPECIAL NOTICE}

Prices quoted herewith cancel all previous quotations, either by letter or in printed lists and are subject to change without notice. All orders taken subject to priority sale, and are F.O.B. our nursery at Des Moines.

TERMS: Cash with order. Remittance may be made by money order, bank draft or check.

SHIPMENTS: We aim to ship all orders the same day received, whenever possible to dig from the field. 


\section{Russell's}

\section{Garden Guide}

In presenting you with our 1932 list, we have selected many new and highly recommended varieties of fine alpines and perennials, to add to our long list of established, dependable plants, and it is our desire to give you the finest and newest varieties at the lowest prices possible.

All our plants are grown in our own fields, and by intensified cultivation, we develop a fine system of roots, that will transplant easily and get established in your gardens very rapidly. Our growing program permits us to send out large, strong plants that will bloom and thrive in your garden.

Our organization while not the oldest, is rich in experience-and having a very large stock of the items we offer-can well claim to set a standard for quality. Everything needed for the proper growth and cultivation is at our command-fertile soils of varied texture-modern scientific propogation methods-unlimited equipment and labor for caring for the growing stock-all go to make Russell's Gardens your sarest, surest source of plant materials.

We are located in a belt where the summers are hot and dry, our winters are severely cold-the finest testing grounds in the country-"What will live and grow for us, will live and grow for you." 


\section{ROCK PLANTS and PERENNIALS}

The true garden lovers, those who are taking a real interest in growing fine plants, are demanding good sized plants, with well established root systems. Russell's plants meet the requirements of the most critical. All orders are fresh dug, with the soil on the delicate rootlets, for it is these tiny roots that make for quick growth in your garden. This assures you of strong healthy plants.

Our prices affords you an opportunity to make an extensive planting at a moderate cost.

The descriptions in our list are meager because of the wide variety of plant material offered. We have given you the blooming season, the color of the blooms as well as the heighth of the plants, these being the most important requirements.

Achillea tomentosa-yellow, 6 in.; Sept.

Agrostema-magenta, 18 inch; August............. .25

Ajuga reptans-dark-blue, 3 inch; May............. $\quad .25$

Alyssum argenteum-yellow, $1 \mathrm{ft}$; May.............. $\quad .25$

ideum-soft yellow, 8 inch; June........................ .35

repens-yellow, 8 inch; May-July....................... $\quad .25$

rostratum-pale yellow, $1 \mathrm{ft}$; J June................. .25

saxatile compactum-yellow, $1 \mathrm{ft}$; May.......... .25

serpyllifolium-yellow, 4 inch; June................ .35

spinosum roseum-rosy-white, 6 inch............... $\quad .50$

Anchusa myosotidflora-blue, $1 \mathrm{ft}$; April......... .35

Anemone pulsatilla-purple, 9 inch; April......... $\quad .35$ pulsatilla rubra-maroon, 9 inch; April........ .35

Aquilegia coerulea-blue and white, $1 \mathrm{ft}$............. .35

dobbies hybrids-soft colors, $1 \mathrm{ft}$...................... 35

flabellatta nana-white, 6 inch; May.............. .35

Mrs. Scott Elliott-soft colors, $1 \mathrm{ft}$................. .25

pyrenaica-dark blue, 6 inch; May, rare....... $\quad .50$

Arabis alpina-white, gray leaves, 8 in............ $\quad .25$

Areneria ceaspitosa-white trailing; May.......... $\quad .25$

Armeria laucheana-rose color; 8 in.; May..... $\quad .35$

Artemesia glacialis-yellow, 6 inch; June.......... $\quad .35$

silver king-white, gray leaves, $2 \mathrm{ft}$................ .25

Aster alpinus-violet blue, 6 in.; June................ $\quad .25$

Aubrietia gracea-violet, trailing; May............. $\quad .35$

Bleeding Heart (See Dicentra)

Calamintha alpina-purple, 6 inch; June........... .25

Campanula allioni-lilac purple, 4 inch; June.... $\quad .35$

carpatica-blue bells, 6 inch; June................. $\quad .25$

garganica-star shaped blue, 4 inch; June.... .35

garganica hirsuta-blue, 4 inch; June............ .35

muralis-deep blue bells, 4 inch; June.......... .35

persicifolia_violet blue, 18 inch; July........... .25

piperi-bright blue, 1 foot, May-July............ $\quad .40$

rotundifolia - clear blue, 1 foot; June........... .25

steveni-violet blue, 8 inch; June; rare........... .50

turbinata - deep blue, 6 inch; June-Aug.......... .25

Candytuft (See Iberis)

Cerasteum tomentosum-white trailing; May.... $\quad .25$

Chives-onion like leaves, lavender, $1 \mathrm{ft}$........... .25

Coreopsis grandiflora-yellow, $1 \mathrm{ft}$; May.......... . .25

Anemone pulsatilla is a gem in the rockery. 
Coral Bells (See Heuchera)

Daisies (See Shasta Daisy)

Daphne cneorum-bright pink, $1 \mathrm{ft}$; May.......... 1.25

Day lilies (See Hemerocallis)

Delphenium belladonna-sky blue, $3 \mathrm{ft}$................. $\quad .25$

bellamosa-dark blue, $3 \mathrm{ft}$; June.................... $\quad .25$

Russell's hybrids-mostly doubles..................... $\quad .35$

Dianthus alpinus-deep rose, 4 in.; June............. $\quad .50$

caesus-bright pink, 6 in.; May-July................ .25

clove pinks-pink, single carnations.................. $\quad .25$

deltoides-bright pink, 6 inch; May.................... $\quad .25$

sundermanni-white, 6 inch; June, rare....... $\quad .50$

Dicentra eximia-pink, all summer, 6 inch......... $\quad .25$

spectabile-heart shaped pink, 18 inch.......... .50

Draba olympica-golden yellow, 4 in.; July........ $\quad .40$

Dryas sundermanni-white, 6 inch; rare......... $\quad .50$

Edelweiss (See Leontopodium)

Erodium amanum-white, 8 in.; May-July; fine .50

Erysimun pulchellum-bright yellow, 6 in......... .25

Flax (See Linum)

Gaillardi-reddish brown, 2 ft.; May-Oct........ .25

Geum alphine borisi-orange scarlet, 6 in.......... $\quad .50$

Geraneum pratense-blue, 2 ft.; June-Aug........ $\quad .25$

sanguineum-red purple, trailing; May........... .25

Grass-ornamental, fine foliage, 8 inch............... .25

Gypsophila paniculata-white, 2 ft.; July............ $\quad .25$

repens-white, trailing; July, very fine............. $\quad .25$

Helenium hoopesi-pure orange, 18 in.; May..... $\quad .25$

Helianthemum hybrids-soft colors, 9 in.......... $\quad .35$

Hemerocallis flava-lemon day lily; June......... $\quad .25$

Dr. Regel-orange day lily, $2 \mathrm{ft}$; May........... .25

early lemon-2 ft., blooms with Iris................ .35

Heuchera sanguineum-coral red, $1 \mathrm{ft}$; May... $\quad .35$

Iberis sempervirens-white, 9 inch; June.......... $\quad .35$ gibraltarica-pinkish white, 6 in.; June........... $\quad .25$

Iris gormanni-soft yellow, 6 in.; very rare....... $\quad .50$ pumilla-white, yellow, purple, 6 inch............. $\quad .25$ sky blue - sky blue, 5 inch; May, fine............... . .25

Lavendula vera-sweet lavender, blue, $1 \mathrm{ft}$......... . 35

Lemon Lily (See Hemerocallis)

Leontopodium alpinum-Edelweiss, 4 inch........ $\quad .35$

Lewisia columbianum-red purple, 8 in.; May.. $\quad .50$ finchi-soft pink, 6 inch; June, rare............ $\quad .50$

Lillium tenifolium-scarlet, 18 inch; June....... . .35

Linaria alpina-blue, 6 inch; July, choice.......... .25

Linum alpinum-sky blue, 4 inch; May-Sept.... $\quad .50$ flavum-yellow cup like, $1 \mathrm{ft}$; June-Aug....... $\quad .35$ perenne-pale blue, 18 inch; May-Oct............ .25

Lychnis alpinus-reddish pink, 4 in.; May....... .25

Monardi didyma-magenta red, $2 \mathrm{ft}$; June..... $\quad .25$

Nepeta mussini-rich blue, 9 inch; May-Sept..... $\quad .25$ wilsoni-purple, 9 inch; May, slow grower... $\quad .25$

Orange Lily (See Hemerocallis)

Oriental Poppy (See Papaver)

Painted Daisy (See Pyrethrum)

Pansies-giant mixed colors, very choice; doz... $\quad .50$

Papaver Mrs. Perry-salmon rose, $2 \mathrm{ft}$.; May.. $\quad .50$ orientalis-brilliant red, $2 \mathrm{ft}$; May.................... $\quad .25$ royal scarlet-dark red, 2 foot; May................. $\quad .50$

Penstomen coccineus-scarlet, 18 inch; July... $\quad .35$

Aubrietias are colorful in the alpine garden. 
Phlox amoena-bright pink, 4 inch; June......... .25

Phlox sublata alba-white, trailing; May........ .25 fairy-light lavender, trailing; April............... .35 lilacina-lavender, trailing; April-June........... .25 rosea-bright pink, trailing; April-June........ .25 vivid-brightest pink, trailing; May.............. $\quad .50$

Phlox B. Comte-French purple, 18 inch; Aug. .25 beacon-bright cherry red, 18 inch; Aug...... .25 enchantress-salmon pink, very choice............ .25 firebrand-brilliant scaret; 18 inch; Aug......... . .25 flora reedy-white, long bloomer; Aug......... .25 milly von hoboken-bright pink, August.......... .25 miss lingard-white, waxy leaf; June.............. .25 rhinelander-salmon pink, red eye; Aug......... .25 rhynstrom-rosy pink, free bloomer; Aug.... .25 R. P. Struthers - Salmon pink, very good........ $\quad .25$ Sir. Edw. Landseer-crimson, 18 inch; Aug. .25 Physostega-bright red bells, 18 inch; Aug.... . .25

Platycodon grandifolia-blue bells, 18 in.; July .25 mariesi-dwarf blue, 9 inch; July, choice....... .35

Plumbago larpentea-purple, 6 inch; Aug.-Oct... .25

Polemonium reptans-blue, 8 inch; April............ .25 carneum-pale rose, 8 inch; June-July............. $\quad .50$

Potentialla doubles-yellow to orange; May...... .35 fruticosa-yellow, 18 inch; shrub.................... $\quad .50$

Primula auricula-dark colors, 6 inch; June...... $\quad .25$ cashmeriana-deep violet, $1 \mathrm{ft}$; May.............. $\quad .50$ cortusoides-deep rose, 10 inch; June............ $\quad .50$ denticulata_deep lilac, 1 ft.; June................. $\quad .50$ veris-orange to salmon, 8 inch; May............ $\quad .35$

Pyrethrum Jas. Kelway-dark red; choice...... $\quad .35$ Russell's hybrids-rose, red and white............ .25

Ranunculus acris-yellow, 18 inch; June.......... .25

Salvia argenteum-cottony down, 6 inch............ $\quad .25$

Saponaria ocymoides-pink, trailing; June...... .25

Saxifraga cordifolia-light pink, 9 inch............. $\quad .35$ McNabiana-white, compact rosettes.............. $\quad .50$

Scabiosa japonica-hardy, blue, $1 \mathrm{ft}$; June...... .25

Sedum acre-green foliage, yellow, 2 inch........ .25 adanotrichum-notched foliage, 4 in.; rare.... .50 album - white, waxy foliage, trailing................ $\quad .25$ altissimum-round bluish-green, 3 inch....... $\quad .35$ amomalum-notched green foliage, 3 inch.... $\quad .35$ anglicum-white flowers, gray green, 3 inch .25 borderi-green fleshy leaf, 8 inch; rare.......... .50 brevifolium - green foliage, 2 inch................... .25 dasyphyllum - fine green foliage, 2 inch.......... .35 ewersi-gray green foliage, 6 in.; good........... $\quad .25$ hispanicum-gray green leaf, 3 inch............... .25 kamschaticum-neat trailing variety .............. $\quad .35$ lydium-bright green leaf, red stems, 2 in..... $\quad .35$ nevi-light green rosettes, extra choice........... .35 pulchellum-bright green, 4 inch; fine............... .35 pruniatum-blue green, 4 inch; choice............... $\quad .35$ reflexum-cocks comb variety, 4 inch.............. $\quad .35$ sarmentosum-yellowish green, trailing ........ $\quad .25$ sexangulare-dark green, yellow flowers......... $\quad .25$ sieboldi-thick gray leaf, 8 inch; good............... .35 spectabile-light green, pink, 1 foot; Aug..... .25 spectabile brilliant-red flowers, $1 \mathrm{ft}$............... . .25 spurium coccineum-good creeper, pink.......... .25 ternatum-neat foliage, 4 inch, new.................. .35

Shasta daisy alaska-white, yellow centers...... .25 hartje \& elder-very early, May, 18 inch...... .25 laucanthemum-double white, 1 foot; May.... .35 
Sempervivum alberti-pale green, 1 inch........... $\quad .50$ alpinum-neat rosettes, $11 / 2$ inch; rare.......... .35 arachnoideum-cob web variety, 1 inch....... .25 arenarium-tiny green rosettes, 1 inch.......... .35 blandum-pale green, 2 inch; red in fall....... .35 browni-green rosettes, red over winter........ .35 doellianum-gray, hairy leaf, 2 inch............ .35 fimbriatum-green rosettes, brown tips.......... .35 fauconette-green, tipped brown, 2 inch....... .35 funcki-light green rosettes, 2 inch; rare..... .35 glaucum-light green, 3 inch; very good....... . .25 globiferum-light green, very compact.......... .25 hirtum-pointed leaves, $11 / 2$ inch; new.......... .35 juratense-rare variety, 2 inch; limited....... . 35 lageri - cob web variety, 2 inch; very good .25 montanum-very tiny green rosettes, 1 inch .35 pallidum-bright green rosettes, 3 inch....... .25 pittoni-dense leaves, green, $11 / 2$ inch............ .35 pyrenaicum-green rosettes, 2 inch; fine...... .25 rubicundum-green leaf, brown tipped........... .75 Russell's hybrid-reddish brown, 5 inch..... 1.50 soboliferum-light green, compact, $2 \frac{1}{2}$ inch .25 tectorum-dark green rosettes, 3 to 4 in...... .25 tectorum hybrids-improved tectorum, 4 in. $\quad .35$

Silene shafta-rosy purple, 4 inch; August..... $\quad .30$

Spirea filapendula-fern like foliage, $1 \mathrm{ft}$....... . 25

Stachys lanata-wooly leaf, 6 inch; fine............. .25

Statice latifolia-lavender, 18 inch; July............ . .25

Sweet williams-bright colors, 9 inch; May....... .25

Thymus serpyllum-creeping thyme, pink, 4 in. $\quad .25$ serpyllum carnea-dense dark green, May... . 35 serpyllum coccineum-bright rose, trailing... .25 lanuginosa-wooly thyme, trailing; choice... .35

Trollius-orange to yellow, 1 foot; May.......... .35

Tunica saxifraga-light pink all summer, 6 in. .25

Verbena venosa-trailing, rose, all summer....... .25 •

Veronica amethystina_rich blue, 9 in.; June..... . 25 corymbosa-azure blue, 6 inch; very rare... $\quad .50$ incana-gray leaf, blue, 6 inch; July............. .25 repens-dark green leaf, blue, 4 inch; June.. $\quad .35$ rupestris-matted creeper, blue, 1 in.; June.. $\quad .35$ rupestris rosea-rose color, 4 inch; June....... .25

Viola johnie-miniature pansies, 6 inch; May.. $\quad .25$ lutea-yellow, 6 inch, all summer.................. .25 pedata - acid loving, purple, 3 inch; June...... . .25 puck-yellow upper, purple lower petals....... . .25 Sutton's apricot-bright apricot, 6 inch......... .25 Yucca-creamy white, 3 foot, showy; 4 year..... . 35

\section{Holland Grown Bulbs}

We import direct from Holand, the highest quality of bulbs and can offer you a long list of Rock Garden bulbs such as Crocus, Scilla, Fritillaria, Grape Hyacinth, Snowdrops, and other early Spring blooming bulbs, as well as the best in Tulips, Hyacinths and the larger bulbs. Prices at the market, which is made up in early summer, according to the crop in Holland. We guarantee you only the finest of bulbs. 


\section{BETTER IRISIS}

Afterglow-lavender buff, 36 in. 2 for

Albert Victor-dark blue, 40 inch; 2 for............ .25

Ambassaduer-brownish red, 48 inch................... .35

Amber-rich yellow, 36 inch................................... .75

Ann Page-blue bicolor, 36 inch; fine..................... .35

Aphrodite-clear pink, 40 inch.............................. 1.50

Archiveque-deep violet-purpie, 2 for................ .25

Argynis-yellow and maroon, 36 inch................ $\quad .50$

Asia-lavender and gold; showy, 40 inch............. .75

Aurea-chrome yellow, 30 inch; 2 for................. $\quad .25$

Autumn King-large lavender, 30 inch................ .35

27 Avril-violet purple, 40 inch.......................... 1.00

Baldwin-violet self, 40 inch; choice.................... 2.00

Ballerine-blue, 40 inch; sweet scented.................. .35

Beau Ideal-violet plicata, 32 inch ......................... $\quad .25$

Benrimo-blue and purple, 42 inch......................... $\quad .35$

Black Prince-velvety purple, 32 inch.................... .35

Bluett-violet purple, 30 inch; early..................... $\quad .25$

Bruno-large purple, 40 inch................................ 3.00

Buto-dark blue, 42 inch; very large................... 3.50

Caprice-wine red, scented; 30 inch; 2 for.......... $\quad .25$

Caroline E. Stringer-rose, 36 inch........................ .35

Cassandra-rose violet, 40 inch............................. $\quad .50$

Church Mouse - coffee brown, 36 inch................... 2.00

Dalila-red purple, 30 inch................................... $\quad .25$

Delicatissima-pink pallida, $3 \mathrm{ft}$.......................... $\quad .50$

Drake-dark blue, 3 ft.; 2 for............................ $\quad .25$

Dream-lilac pink, 3 foot.................................... $\quad .25$

Duke of York - sky blue, very large, 36 inch...... $\quad .25$

Doxa-olive green, 30 inch, rare........................ 3.50

Eldorado-bronzy yellow, 3 ft.; 2 for................. .25

Euphony-mustard yellow, choice; 36 inch...... 1.00

Fairy - white, scented, 40 inch; 2 for.................. .25

Frammeschwert-yellow and brown, 36 inch.. .35

Frieda Mohr-lilac rose, 36 inch......................... 2.50

Gertrude-early dark purple, 30 inch; 2 for.... $\quad .25$

Gold Crest-violet blue, 30 inch; 2 for................. .25

Harmony-purple blue self, 36 inch.................... $\quad .35$

Her Majesty-rose pink, 30 inch; 2 for............ .25

Hermosa-bright pink, 36 inch; 2 for.................. $\quad .25$

Isoline-old rose, 36 inch......................................... $\quad .25$

Jubilee-buff plicata, 3 foot.................................... .25

Jumbo-violet purple, 3 foot................................. 1.00

Juniata_blue violet, 40 inch; 2 for........................ $\quad .25$

King Karl-reddish brown, 3 foot......................... $\quad .25$

King Tut-rich dark red, 30 inch........................ 1.75

Kochi-velvety purple, 30 inch: 2 for.................. .25

King Juba-red purple, 42 inch............................ 3.00

Lent A. Williamson-violet purple........................ $\quad .25$

Leonadas-lavender purple; 2 for........................... $\quad .25$

Lepinoux-deep purple, 48 inch.............................. 1.00

Lohengrin-silvery lilac; 2 for.............................. $\quad .25$

Lona-buff plicata, 30 inch; pleasing.................. $\quad .25$

Lord of June-soft blue, 42 inch......................... $\quad .25$

Loreley-yellow and purple, 30 in.; 2 for............ .25

Labor-dark red, 40 inch ..................................... 2.00

Madam Chereau-white, $40 \mathrm{inch} ; 2$ for................ $\quad .25$

Madam Chobaut-buff. 3 foot; 2 for.................... .25

Magnifica-violet purple; 4 foot............................. .35

Ma Mie-white, 36 inch: 2 for................................. .25

Mentor-rich purple, 36 inch................................. .25

Midwest-ruffled purple plicata............................. $\quad .25$

Midgard-pink and vellow blend; choice........... 1.50

Mildred Presby-white and violet, 30 inch......... .25

Mme. Paquette-rose claret, 40 inch..................... .25

Monsignor-deep purple, 36 inch; 2 for................. .25 
Majestic-purple, 36 inch................................ $\quad .50$

Morning Splendor-red purple, 48 inch.............. $\quad .50$

Mother of Pearl-sky blue, 40 in.; 2 for......... .25

Mrs. Alan Gray-pinkish lilac; 2 for................. .25

Mrs. Haw-pink, 36 inch; 2 for....................... .25

Mrs. Wicht-yellow and maroon....................... 1.00

Marsh Marigold-yellow and brown................ .25

Nebraska-apricot yellow and brown............... 2.00

Nibelungen-fawn and purple; 2 for................ .25

Nine Wells-velvety purple; 2 for.................... . .25

Nuee D'Orange-maroon, 36 inch....................... .25

Old Gold-old gold, 36 inch.............................. 1.00

Omaha-purple, 36 inch, large........................ 2.50

Opera-red purple, 36 inch............................... .25

Otoe-red, large and fine, 36 inch........................ 2.00

Parc De Neuilly-blue, $3 \mathrm{ft}$; 2 for.................... . .25

’arisiana-purple plicata; 2 for........................ . .25

Prairie Gold-yellow self, 36 inch.................... .75

Princess Beatrice-clear blue; 2 for............... . 25

Princess Lohengrin-silvery lilac ....................... $\quad .75$

Princess Royal-violet blue, 3 ft.; 2 for............. .25

Princess Victoria Louise-yellow; 2 for........... .25

Prospero-large red purple, 42 inch.................... .25

Prosper Laugier-brown, 36 inch; 2 for............ .25

Purple Haze-gray self, 32 inch....................... 1.25

Purple King-red purple, 30 inch; 2 for............ .25

Quaker Lady-smoky lavender, 3 ft.; 2 for..... $\quad .25$

Queen Caterina-lavender, 42 inch..................... $\quad .25$

Queen of the Gypsies-old bronze; 2 for........... .25

Queen of May-rose lilac, 30 inch; 2 for.......... .25

Red Cloud-red lilac, 30 inch; 2 for................... .25

Rhine Nixe-purple, 36 inch; 2 for................... . .25

Rose Unique-early pink, 3 inch; 2 for............. .25

Roseway-purple, 36 inch; 2 for..................... .25

Seminole-velvety red, 3 foot........................ .25

Shekinah-lemon yellow, 36 inch...................... .25

Sherwin Wright-yellow, 30 inch; 2 for......... .25

Shrewsbury-rosy bronze, 30 inch.................. .25

Souviner De Mme. Gaudichau-purple self...... . 35

Sunset-coppery yellow, 36 inch...................... .35

Susan Bliss-rose pink, 40 inch........................ .50

Sweet Lavender-lavender, $3 \mathrm{ft}$. ; 2 for................ .25

Tom Tit-violet blue, 18 inch; 2 for................. .25

Trostringer-soft pink, 36 inch......................... 1.00

True Charm-white plicata, 42 inch................. .35

Vioalacea Grandiflora-blue, 3 ft.; 2 for......... .25

Warrior-large purple, 42 inch......................... .50

White Knight-white, 30 inch; late................... . .25

White Queen-pure white, 36 inch....................... .25

Wyomissing-rose, 30 inch; 2 for...................... .25

Zua-crinkled blue white, 18 inch; May............ . .25

Zwannenberg-olive green novelty, 18 inch....... .25

\section{Beardless Irises}

Distinction-blue flowers, 36 inch...................... .25

Emperor (Siberica) - deep violet, 30 inch........ .50

Lord Woolsey-violet and yellow, 40 inch......... 1.00

Orientalis Blue King-deep purple, 30 inch........ .25

Orientalis Snow Queen-snowy-white, 30 inch.. .25

Perry's Blue (Siberica) - sky-blue, 45 inch........ .35

Pseudacorus-yellow English Iris, 40 inch.......... .25

Primula-dwarf iris, white, yellow \& purple, 4-6 inch; 2 for.................................................. .2.

Sky Blue-dwarf, sky blue, 6 inch........................ . .25 


\section{Recent Iris Introductions}

We offer you a selected list of really fine, new introductions of New, Rare Irises, for those that want the best. Delivery after July 15th-good healthy rhizomes, that will bloom for you next year.

Prices are for Prepaid Parcels Post.

ALCHEMY - created much comment in the Taft garden the last three years, undoubtedly a fine yellow. The stems are tall, well branched and bear a good number of solid, deep yellow blooms. Mighty fine. Each $\$ 42.50$.

CLARA NOYES-a favorite of all visitors at our gardens last year. The coloring is a medley of peach and apricot tones, suggesting the blends of the rose "Talisman." Stock limited, as every one wants it at sight. Each $\$ 9.50$.

DAUNTLESS-appears much redder than any of the other so-called red ones. The blooms are of great size, on tall stems and flowers over a long season. Each $\$ 10.00$.

DEPUTE NOMBLOT-the worlds greatest iris. Bloomed for the first time in America in 1930, and it took every one by storm, that saw it. Standards light rosy-purple, overlaid bronzy-gold. Falls very wide, purplish garnet-red, deep orange beard. Over four feet tall, wonderfully branched. Each $\$ 25.00$.

DOLLY MADISON-a wonderful blending of colors, a huge Quaker Lady this variety has no superior in its class. It is reliable everywhere, and is admired by all that see it. Each $\$ 2.75$.

INDIAN CHIEF-Well named, being regarded as a red blend. The falls are deep blood-red, blended with bronze and the standards are lighter in tone. A large flower, well branched, tall stems. Each $\$ 7.50$. MELCHOIR-The deep velvety-maroon flowers leave nothing to ask for in size, shape or all round Iris perfection. It is a self of velvety-garnet, with beard of bronze-yellow; distinctly different. Each $\$ 7.50$.

MIDWEST PRIDE-a wonderful variety by Hans Sass, with rich coloring. Standards are violet, and the falls are a rich deep violet-purple. Resembles Archiveque, but is very much larger, on tall stems. Certain to be much in demand. Each $\$ 10.00$.

RAMESES-another winner by Hans Sass, which went over big with us last year. Standards are light russett, with a yellow glow through the center. Falls pink, with prominent orange beard. Very hardy and vigorous, one of the fine new blends. 36 inch. Each \$8.00.

RED DOMINION-a pretty garnet-red Dominion, introduced last year. While the standards are a shade lighter than the falls, it is practically a self in effect $-a$ piece of rich red velvet. The buds are verv striking. Each $\$ 27.50$.

WAMBLISKA - This huge white self, by Jacob Sass was the sensation of 1930 . Few Irises are larger, and it is sure to enjoy a wide popularity for many a season. Extra choice. Each $\$ 15.00$.

W. R. DYKES-here is a yellow iris that ranks with the largest in size, with heavy creepy texture and a color of richest golden-yellow. It has flowered in almost everv section of the country. A great favorite. Each $\$ 23.00$.

ZUNI-a glorious glowing red-brown, overlaid with a coppery suffusion. Falls are a brilliant red and the standards seem to be sprinkled with gold dust. Large size. well branched and about 40 inches in heighth. Each $\$ 12.50$. 


\section{Newer Water Lilies}

We know that you will enjoy these varieties as we propagate only those that have proven to be the most popular. They are selected because they are prolific bloomers and sturdy growers.

HARDY WATER LILIES

Attraction-Deep red, large blooms....................\$5.00

Comanche-amber red, prolific, choice.................. 3.50

Conquerer-dark red, stripped white....................... 3.00

Escarboucle-bright red, very fine.......................... 5.00

Nympheas Pink-soft pink, free bloomer.......... 1.50

Nympheas Yellow-fine yellow, free bloomer.... 2.00

Sunrise-large, soft yellow; new.......................... 5.00

Yellow Pigmy - for tubs or small pools................. 1.25

\section{TROPICAL LILIES}

Geo. Huster - red night bloomer.......................... $\$ 2.50$

Blue Triumph-large dark blue, prolific............. 3.00

Blue Star-blue, very fragrant, fine.................... 2.50

Rose Star-rose pink, very fragrant....................... 2... 2.50

Purple Wonder-rosy purple, new and fine........... 5.00

\section{SACRED LOTUS}

Sacred Lotus-fine pink flowers...........................\$2.50

\section{WATER PLANTS}

Water Lettuce-10c each, 3 for.............................25c

Water Hyacinth-10c each, 3 for.............................25c

Water Poppy-20c each, 3 for.................................50c

Parrot Feather-10c each, 3 for................................25c

\section{Peonies}

(3 to 5 Strong Eye Divisions)

Adclph Rousseau-dark red, early. $\$ 1.00$

Chestine Gowdy-pink, midseason.

Edulis Suberba-deep pink, early........................... $\quad .50$

Felix Crousse - ruby red, early.............................. $\quad .50$

Festiva Maxima-white, early.............................. $\quad .50$

Fontenelle-bright red, early...................................... .75

Grover Cleveland-dark red, late........................ 1.00

Karl Rosefield-rich red, early................................ 1.00

La France-apple blossom pink.............................. 2.75

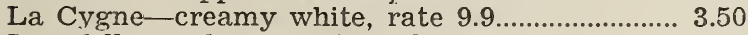

Longfellow-cherry red, midseason....................... 1.50

Mme. Calot_pale pink, early ................................. $\quad .50$

Martha Bullock-large shell pink........................ 3.00

Mary Brand-fine red, early .................................. 1.50

Mons Jules Elie-bright pink, early..................... $\quad .75$

Mikado_-red single Jap, early .............................. 1.50

Mons Krelage - currant red, late............................ 1.00

Mons Martin Cahuzac-dark red......................... 2.00

Proebe Carey - soft pink, late................................... 2... 2.50

Primavere-a near yellow, early............................ 1.75

Richard Carvel-fragrant, red, early.................. 2.25

Old Silver Tip-pink with silver tip........................ $\quad .50$

Rubra Suberba-dark red, late............................. $\quad .50$

Sarah Bernhardt-clear pink................................... 1.00

Solange-white, very fine......................................... 3.00

Therese-bright pink, very fine............................ 2. 2.00

Tourangelle-flesh color, early ................................ 2.00

Arbor Vitea Primadalis, 18-24 inch.......................\$2.50 


\section{EVERGREENS}

There is nothing that has an all year beauty like evergreens, and while they are coming into their own, yet they should be used more, as they need no special care, such as mulching and protecting over winter.

All come to you with the dirt on the roots, baled and burlaped, without extra charge.

Austrian Pine, 2-3 foot............................................. 2.50

Balsam Fir, 2-3 foot.................................................. 3.00

Balsam Fir, 3-4 foot.................................................... 3.75

Black Hill Spruce, 18-24 inch.............................. 1.75

Black Hill Spruce, 2-3 foot................................... 2.75

Colorado Blue Spruce, 18-24 inch............................. 5.75

Colorado Blue Spruce, 2-3 foot.............................. 7.75

Concolor Fir, 12-18 inch....................................... 2.75

Concolor Fir, 18-24 inch....................................... 3.75

Chinese Juniper, 3-4 foot.......................................... 3.25

Chinese Juniper, 4-5 foot..................................... 4.50

Irish Juniper, 18-24 inch..................................... 2.25

Japanese Yew, 12-18 inch................................. 3.25

Juniper Cummunis, 1-11/2 foot spread.................. 3.75

Juniper Japonica, 1-11/2 foot spread..................... 3.00

Juniper Pfitzer, 18-24 inch spread......................... 3.00

Juniper Pfitzer, 12-18 inch spread.......................... 2.00

Juniper Sabina, 12-18 inch spread......................... 2.75

Juniper Sabina, 18-24 inch spread........................ 4.00

Mugho Pine, 15-18 inch spread............................. 2.25

Meyeri Juniper, 15-18 inch................................... 4.00

Scopulorum, 2-3 foot ........................................... 3.25

Siberian Arbor Vitea, 2-3 foot............................... 3.00

\section{Ornamental Shrubs}

We list but a few of the shrubs and trees that are generally used, but we can supply you with most any dependable variety that would be practical in your climate, also can quote you on larger sizes of many trees and shrubs. Your correspondence is invited, and quantities will be gladly quoted.

Fruit and ornamental trees supplied in a good many sizes at rock bottom prices.

Barberry, 21/2-3 foot stocky.................................\$ .50

Barberry, red leaf, 18-24 inch............................... .50

Flowering Almond, pink or white............................ $\quad .50$

Highbush Cranberry, 2-3 foot................................... $\quad .45$

Honeysuckle, Tartarian, 2-3 foot........................... .35

Honeysuckle, Tartarian, 3-4 foot............................. $\quad .50$

Hydrangea, A. G. Early, 18-24 inch....................... .35

Hydrangea, P. G. Late, 2-3 foot............................ .40

Syringa Virginalis, $2-3$ foot.................................... .65

Syringa Virginalis, $3-4$ foot.................................... $\quad .85$

Quince, Japanese, 2-3 foot.................................... $\quad .40$

Lilac, 20 varieties; all colors; $2-3 \mathrm{ft}$.................... .75

Lilacs, 20 varieties; all colors; $3-4 \mathrm{ft}$..................... 1.00

Spirea Anthony Waterer, 18-24 in..................... $\quad .50$

Spirea Froebeli, 2-21/2 foot..................................... .45

Spirea Van Houtti, (Bridal Wreath) 2-3 ft....... .25

Spirea Van Houtti, 3-4 foot...................................... .40

Tamarix, 3-4 foot..................................................... .50

Weigelia, Rosea; 2-3 foot.......................................... $\quad .40$ 


\section{Shrubs for Hedges}

Amoor River Privet, 18-24 inch; 100 for. $\$ 7.50$

Barberry, 12-18 inch; 100 for..................................11.00

Spirea, 18-24 inch; 100 for........................................13.50

\section{Roses}

Hybrid Tea Roses at the lowest prices in years. All plants offered are two-year field grown bushes, special price 3 for $\$ 1.00$.

E. C. Hill-scarlet

Grus An Teplitz-red

Los Angeles-apricot

Joanna Hill-yellow

Red Radiance-deep rose

Radiance-soft pink

Sunburst-apricot

Baby Rambler Roses, 3 for

Taisman-deep pink

Climbing Roses, American Beauty, Pauls Scar-

let, 3 for. $\$ 1.00$

\section{Fertilizer}

BONE MEAL

Analysis: 2\% Ammonia, 2\% Nitrogen, Phosphoric Acid 20\%. An excellent fertilizer, finely ground to act quickly. Free from acid. For lawns apply 50 pounds to 1,000 square feet. Should be used regularly on your plants to increase growth and to intensify the color of the blooms. 5 lbs., 55c; $10 \mathrm{lbs}$., 90c; 25 lbs., $\$ 1.50$; 50 lbs., 2.50; 100 lbs., 3.75. F.O.B. Des Moines.

\section{SHEEP MANURE}

Supplies all the necessary plant food elements. This is a pure natural manure, and its effect is lasting. Fine for mixing with soil to supply humus which is needed in your garclens. For liquid manure, dissolve one pound in five gallons of water. You can use it either liquid or dry form right up to the plants with safety, as it will not burn the plants. We highly recommend it to our customers. $5 \mathrm{lbs}$., 35c; 10 lbs., 60c; 25 lbs., $\$ 1.25 ; 50$ lbs., $\$ 1.90 ; 100$ lbs., \$2.85. F.O.B. Des Moines.

\section{GRANULATED PEAT MOSS}

This is a moisture-retaining humus, which is very important in keeping soils loose and friable, better than manure for the rock gardens. Keeps the soil from crusting and helps prevent fungus growth. 50c per package. F.O.B. Des Moines.

\section{GARDEN FERTILIZER}

Analysis: $3 \%$ nitrogen, 12\% phosphoric acid, 4\% potash. This mixture differs from other balanced plant rations in that it is composed of plant foods in mineral form. The advantage of mineral form is the absence of odor. This is an excellent mixture for the garden, but do not put on the green foliage. 5 lbs., 50c; 10 lbs., 90c; 25 lbs., $\$ 1.95$. 


\section{BOOKS}

\section{MODERN BOOKS FOR YOUR LIBRARY}

We offer practical books, published by that reliable firm of A. T. De La Mare Company, written in understandable language. All prices are postpaid. Just send us your order and books will be sent promptly.

1001 GARDEN QUESTIONS ANSWERED. (By Alfred Hottes). 320 pages, answering our problems. Cloth bound. Postpaid $\$ 1.65$.

ROCK GARDEN PRIMER. (By Archie Thorton). 153 pages, 70 illustrations. All you wish to know about Rock Gardening. Postpaid \$2.15.

BOOK OF ANNUALS. (By Alfred Hottes). 182 pages, 155 illustrations, dealing with annuals. Cloth bound. Postpaid $\$ 1.65$.

BOOK OF PERENNIALS. (By Alfred Hottes). Treats over 125 species, giving heighth, color and blooming time. Cloth bound. Postpaid \$1.65.

GARDEN GUIDE. (Fifth Edition). Covers every phase of pruning, propogating, flowers, shrubs, bulbs, etc. Cloth bound. Postpaid $\$ 1.65$.

LANDSCAPING THE SMALL HOME. (By Edw. Olver). 160 pages of practical pointers on beautifying. Cloth bound. Postpaid \$2.15.

PRACTICAL PLANT PROPOGATION. (By AIfred Hottes). Told in understanding language, how to make cuttings, sow seeds, etc. Cloth bound. Postpaid \$2.15.

BOOK OF SHRUBS. (By Alfred Hottes). 448 pages about better shrubs. 180 halftones and diagrams. Cloth bound. Postpaid $\$ 3.15$.

ROSE GARDEN PRIMER. (By Eber Holmes). 224 pages about rose growing, spraying, etc. 70 illustrations. Cloth bound. Postpaid \$1.90.

WOMAN'S FLOWER GARDEN. (By Jane Leslie Kift). 160 pages of live information about flowers and plants. Cloth bound. Postpaid \$1.35.

WATER GARDENS AND GOLD FISH. (By Sawyer \& Perkins). All about Water Plants, Goldfish, their breeding, diseases and remedies, 112 pages. Cloth bound. Postpaid \$1.65.

BOOK OF WATER GARDENING. (By Peter Bisset). Full details about water gardening, 125 halftones. Postpaid $\$ 5.00$.

Many other practical books on all garden subjects, from which beginner or seasoned grower will benefit by. We have prepared a 32-page booklet, describing almost 50 modern books. It's free for the asking.

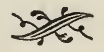

We have many varieties in too small a quantity to list and if there is some plant that you are looking for and do not find it in our list, write us. We may have it, but if not we will be very happy to get it for you, if we possibly can. 


\section{Plants for Walls}

Achilleas
Alyssums
Androsace
Areneria
Armeria
Aubrietia
Campanula Garganica
Carpatica, Muralis
Piperi, Steveni
Dianthus

Achilleas

Areneria

Armeria

Carpatica, Muralis

Dianthus

\author{
Draba \\ Dryas \\ Erodium \\ Iberis \\ Primula Auricula \\ Saponaria \\ Sedums \\ Thymus \\ Tunica \\ Veronica Rupestris
}

\section{For Shady Places}

Ajuga Reptans

Anchusa Mysotidifiora

Aquilegia (partial)

Arenerias

Aster Alpinus

Campanula Garganica

Muralis, Piperi and

Steveni

Dicentra Eximia
Erica Carnea

Erodium Amanum

Heuchera

Phlox Sublata

Primulas

Platycodon

Saxifraga

Veronica

Viola

\section{Vaughn Seeds}

We are pleased to announce that we carry a full line of the choicer Vaughan seeds for the convenience of our customers. There are no better seeds and are sold at popular prices.

Gray foliage plants should be used more, to accent certain plants or spots in the Rock Garden. Bear in mind that all plants that have gray leaves do best in a high dry location, with full sun.

Most plants that have blue flowers like a shady location, the bluer the flower, the more shade they require.

In planting bulbs in the Rock Garden such as Crocus, Scilla, Fritillaria and other early Spring blooming varieties, we like to plant Sedums, Thymus, Veronica Rupestris or other low, matting varieties over them. Bulbs come up through them and after the bulb foliage dies down, an empty spot is avoided. This is a lovely combination. 
Quality Stock at Lower Prices

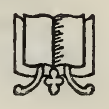

Let us quote on your list of wants.On many items in quantity we can make lower prices than those printed.

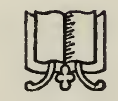

Meet Your Garden Friends Here 